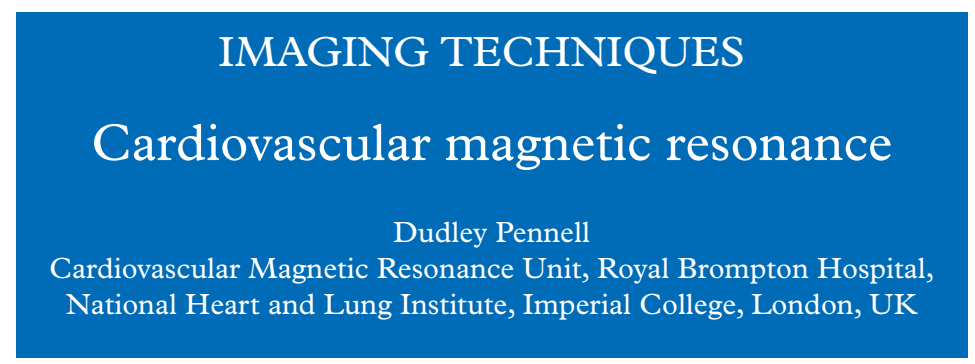

ardiovascular magnetic resonance $(\mathrm{CMR})$ is a new field in cardiology. CMR has enormous potential because of its major attributes of high image quality and resolution combined with non-ionising radiation and versatility. With recent major technological advances there has been a quantum leap in acquisition speed and image quality that makes its use in ischaemic heart disease robust and clinically valuable. This review will focus on the current clinical indications for CMR, starting with those that are well established, and moving on to the developing indications that are expected to reach clinical maturity soon; finally, the less advanced uses of CMR will be briefly summarised.

\section{Fundamentals of cardiovascular} magnetic resonance

What is magnetic resonance?

Magnetic resonance (MR) is a fundamental property of some elements which contain an uneven number of nucleons (protons plus neutrons in the nucleus), and was first described in 1946. These atoms have a property known as net spin, and they absorb radio waves at a resonant frequency which is linearly related to the ambient magnetic field. Nearly all CMR is currently performed at the resonant frequency of hydrogen $(63 \mathrm{MHz}$ at 1.5 Tesla) because it is abundant and yields high signal. However, other nuclei can also be interrogated: phosphorus-31, carbon-13, sodium-23, potassium-39, and fluorine-19. These nuclei give lower signal and usually are only examined using MR spectroscopy, a technique which shows the spectrum of the radio signal. Important results in cardiology have been shown using P-31 MR spectroscopy. ${ }^{1}$

For clinical MR, a process of image acquisition occurs which has some parallel with echocardiography. Energy is transmitted into the heart (radio waves), and after a delay an echo (radio waves) is received. In the intervening period, the hydrogen nucleus becomes excited, and subsequently relaxes. The rate of relaxation is defined by two parameters known as T1 and $\mathrm{T} 2$, and these can be manipulated to give varying contrast allowing tissue characterisation. Many other parameters also affect the signal, such as temperature and oxygen concentration and flow, and these therefore can also be measured using MR. Transmission and reception of the radio waves is achieved using special aerials known as coils. Conversion of the raw
CMR Unit, Royal Brompton Hospital, Sydney Street, London SW3 6NP, UK

d.pennel1@ic.ac.uk data into images requires fast computers and Fourier transformation.

What is cardiovascular magnetic resonance? There are essentially three types of imaging sequence which are used in the cardiovascular system:

- spin echo imaging - the blood is black and good quality anatomical imaging can be obtained;

- gradient echo imaging - the blood is white and high quality cine imaging can be obtained, which is used to identify regional myocardial function and abnormal flow patterns;

- velocity mapping - a variant of gradient echo imaging that in its usual form behaves like two dimensional Doppler, but unlike Doppler it can measure flow directly and can be extended into seven dimensional flow imaging for complex flow dynamics problems. $^{2}$

CMR therefore consists of applications of these sequences and their variants, which allows determination of cardiac physiology, anatomy, metabolism, tissue characterisation, and vascular angiography

What is different about CMR compared with normal MR?

A CMR scanner is a large superconducting magnet with its associated radio wave and computer systems. For dedicated CMR, the environment typically also incorporates medical gases, full invasive and non-invasive physiological monitoring telemetry, stress infusion pumps for adenosine and dobutamine, a power injector for contrast studies, and full resuscitation equipment and drugs. Experience has shown that acutely ill and anaesthetised patients can be safely managed within the magnet in experienced centres. State of the art CMR systems are differentiating from standard MR systems in much the same way as echocardiography systems have become specialised compared with general ultrasound systems used for obstetrics and gynaecology, for example. Modern CMR scanners incorporate ultrafast technology which allows real time imaging (up to 50 frames per second ungated), and ultrafast applications for coronary artery disease. Currently most scans are still gated to the ECG, and in some cases also to the respiratory cycle using advanced MR diaphragm monitoring techniques called navigators.

\section{Safety of CMR}

CMR is safe, and this marks it out from $x$ ray based techniques. MR contrast agents are not nephrotoxic and are much safer than the $x$ ray agents. It is safe to scan all prosthetic heart valves, and patients with sternal wires, joint replacements, and retained epicardial pacing leads. There is abundant evidence that stents are safe to scan any time after insertion. ${ }^{3}$ Pacemakers are problematic, although recent experience at 0.5 Tesla is encouraging, but this should only be considered in centres of specialist experience. Other implantable devices including defibrillators and cerebrovascular aneurysm clips are currently a contraindication to CMR. Claustrophobia occurs in about 
$4 \%$ of patients but this frequently responds to low dose diazepam, to leave a hard core of refusing patients of approximately $1 \%{ }^{4}$

\section{Current established clinical indications}

\section{Aorta}

As a large conduit, the aorta is easily investigated by CMR for its entire length in the thorax. Three point plane definition techniques are useful for imaging in the long axis of the aorta as it curves out of the coronal and sagittal planes. Typically, points are taken in the ascending and descending limbs and the arch to achieve this. The hockey stick view so obtained is ideal for showing the extent of dissections and the location of coarctation. Closer interrogation of specific regions can also be made with the orthogonal planes. CMR has been shown in randomised trials to be more accurate than transoesophageal echocardiography (TOE) and computed tomography in acute dissection, ${ }^{5}$ although rapid access to echocardiography is simpler to organise in most hospitals. CMR is preferred for chronic follow up of these patients, in order to exclude aneurysm formation at the margins of the operation site, and other complications. In coarctation, the location of stenosis frequently makes Doppler problematic, and CMR is ideal in answering clinical issues, as well as being cost effective (fig 1). ${ }^{6}$ CMR can also demonstrate the net flow in collaterals as an index of stenosis severity.

\section{Congenital heart disease}

Echocardiography is excellent in defining congenital disease in the young, but with growth into adulthood and after corrective surgery, CMR plays a larger role and is often complementary to TOE. Studies show that TOE is better at defining fine structure such as valve morphology, whereas CMR is better at showing flow, conduits, and great vessel anatomy. In centres with expertise in both techniques, invasive catheterisation has greatly reduced in frequency, being required mainly for pressure measurements.

\section{Angiography}

For angiography of all vessels (arterial and venous) other than the coronary tree, CMR is rapidly becoming the clinical test of choice (fig 2). MR angiography is fast, simple, and safe, requiring only a peripheral intravenous injection of paramagnetic gadolinium contrast agent without catheterisation, and 4-20 seconds of three dimensional acquisition, according to scanner capability. The data can be displayed in a three dimensional rotating cine, and on the latest scanners, such cines can also be time resolved (four dimensional angiography). This has proved useful for the pulmonary arteries. Major application has been shown for the aorta, renal, and leg arteries, ${ }^{8}$ but the technique is not limited to these areas. Recently thrombus imaging with CMR has been shown to be very sensitive in the venous system, ${ }^{9}$ and comparisons with established techniques for detection of deep vein thrombosis and pulmonary embolism are under way.

\section{Masses and tumours}

CMR is ideal for defining the size, extent, and relations of masses to surrounding tissues

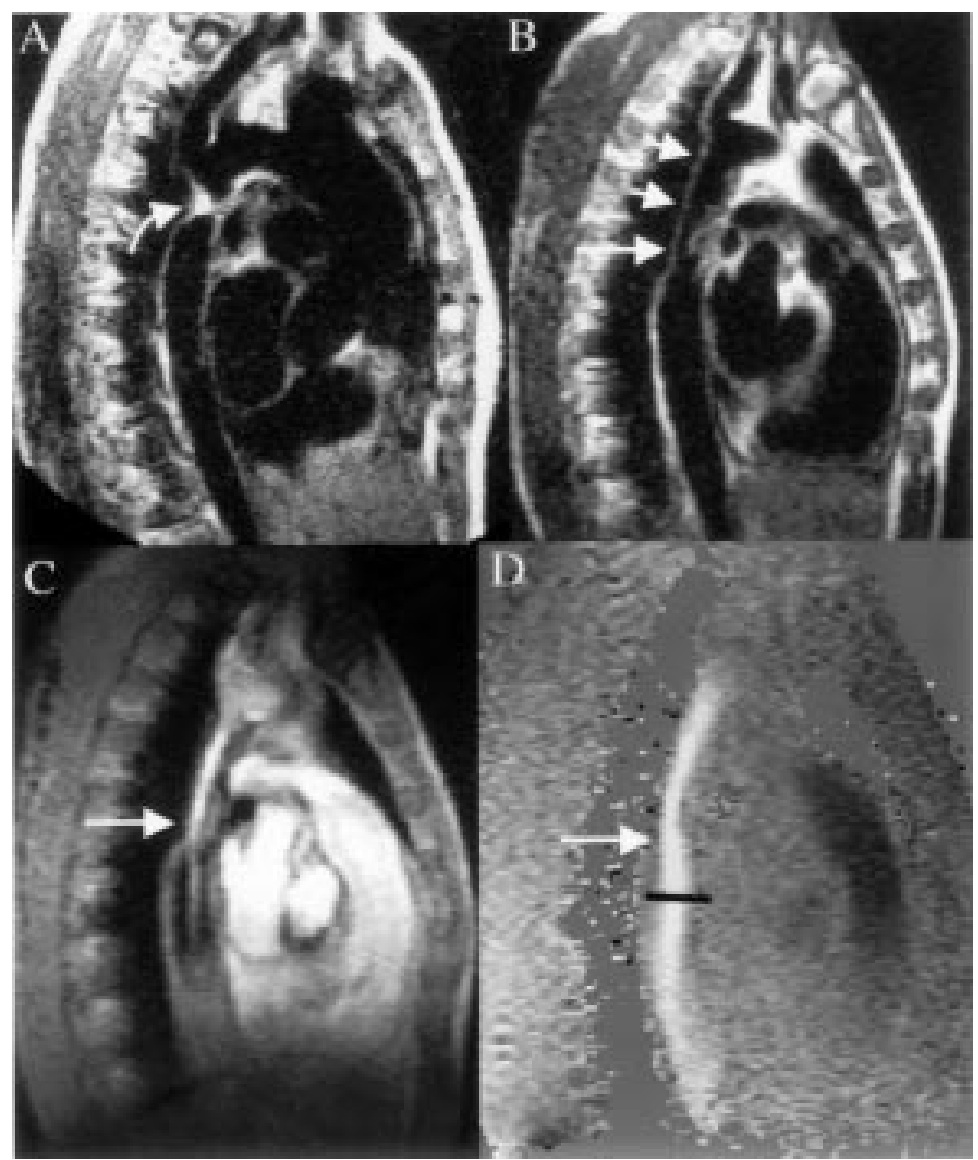

Figure 1. CMR in a patient with coarctation. (A) The preoperative spin echo image in an oblique sagittal plane shows the entire thoracic length of the aorta, and the coarctation (curved arrow) just distal to an enlarged left subclavian artery.

(B) Appearance of the same region with spin echo imaging some years after repair of the coarctation site with a Dacron graft (short arrows). There is narrowing of the distal end of the graft and this is clearly seen in $C$ with the systolic frame of the gradient echo cine showing bright signal within the graft from increased velocities and flow enhancement. Immediately distal to the graft narrowing (straight arrow) a bright jet is seen exiting into the normal descending aorta surrounded by dark areas which are caused by signal loss from turbulence. The velocity map D, from exactly the same plane as $\mathrm{C}$, shows intense white colouration and a peak velocity measured at $3 \mathrm{~m} / \mathrm{s}$ ( $36 \mathrm{~mm} \mathrm{Hg}$ pressure gradient).

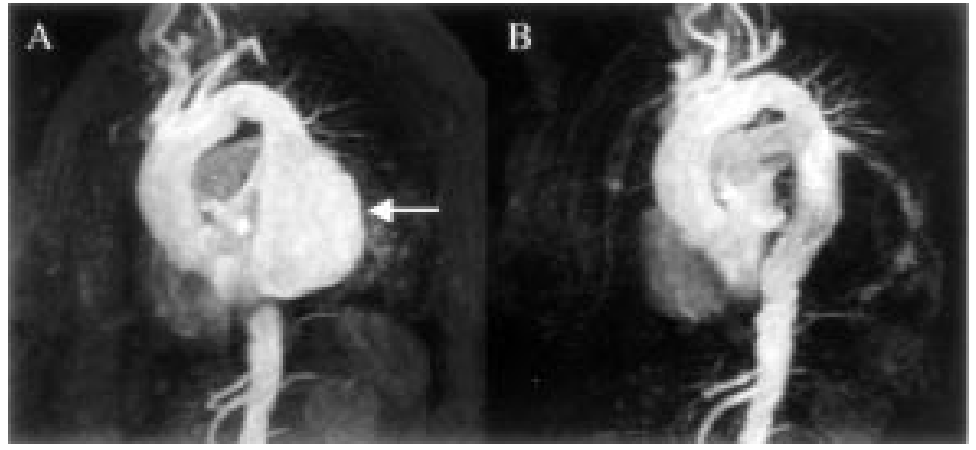

Figure 2. CMR three dimensional angiography of a patient with a descending aortic aneurysm (arrow) before (A), and after (B) insertion of a covered stent. The technique involves a peripheral injection of gadolinium contrast agent and data acquisition for eight seconds. Each of the images shown is one from a three dimensional data set which can be rotated to appreciate fully the anatomy, and plan the length and size of stent for this procedure. Reproduced from Stables et al, Circulation 2000;101:1888-9, with permission of the publisher. 


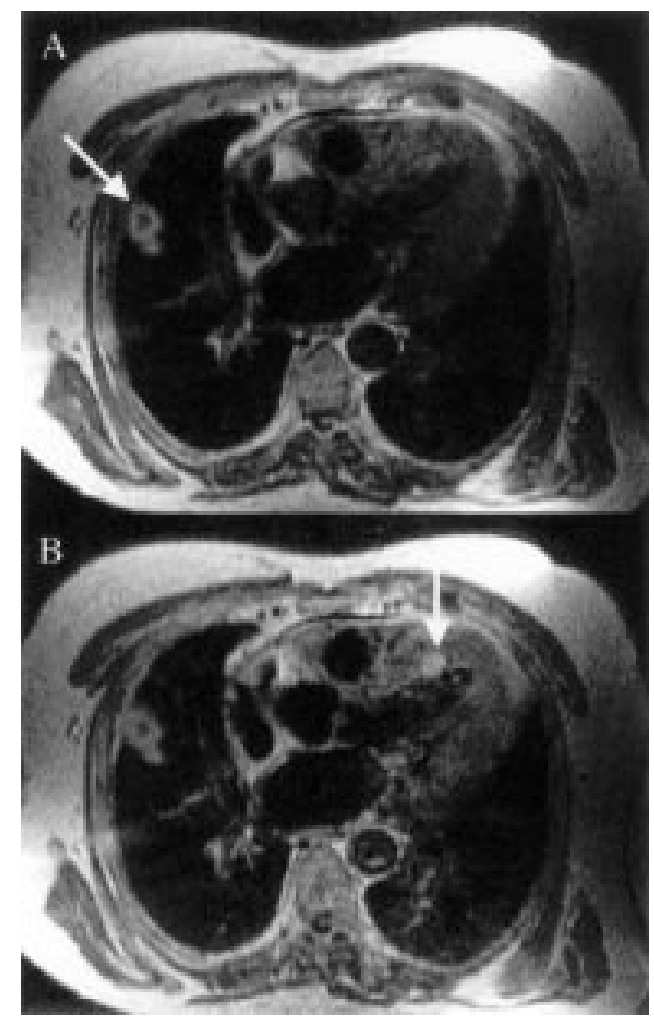

Figure 3. CMR before (A) and after (B) gadolinium enhancement in a patient with an abnormal chest $x$ ray, and symptomatic ventricular premature beats. After gadolinium a clear enhancing lesion is seen in the subendocardium of the apical septum (vertical arrow). This was a metastatic chondrosarcoma from a chest primary (angled arrow).

because of the high quality of the three dimensional imaging. ${ }^{10}$ There are two other techniques which make CMR useful for assessment of cardiac masses, namely tissue characterisation and the presence of enhancement with gadolinium contrast. $\mathrm{T} 1$ and $\mathrm{T} 2$ weighted images vary between masses according to their biochemical composition, and this can be used to distinguish between them. For example,

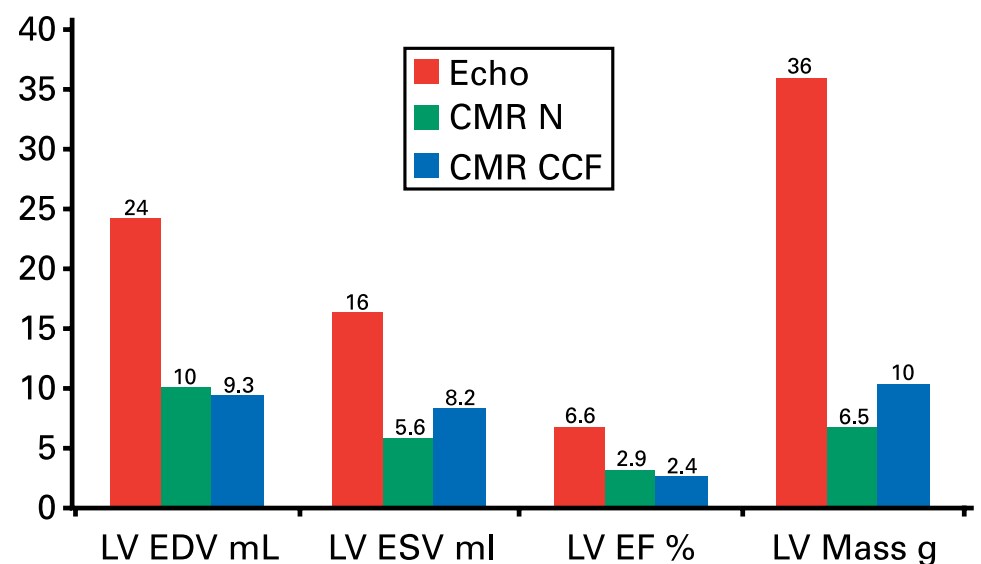

Figure 4. Comparison of the standard deviation of inter-study reproducibility between echocardiography and CMR. This standard deviation is central to determining sample size for drug trials. Sample size rises with the square of the ratio in standard deviations between the techniques. For all remodelling parameters of function and mass, the variability of two dimensional echocardiography is greater. The comparative figures come from different patient groups-echocardiography data from Otterstad et al, Eur Heart J 1997;18:507-13, and CMR data from Bellenger et al. ${ }^{11} \mathrm{~N}$, normals; CCF, congestive cardiac failure; LV, left ventricle; EDV, end diastolic volume; ESV, end systolic volume; EF, ejection fraction. pericardial cysts have very characteristic high signal on T2 imaging. In addition, the fat content of tumours can be selectively imaged with the technique of fat suppression. Gadolinium enhancement reflects tumour vascularity and therefore positive enhancement occurs in malignancy (fig 3). Some capsular enhancement is expected in benign tumours as well, however, and vascular benign tumours such as myxoma also enhance. Overall, however, these additional characterisation features are very useful clinically for guidance for diagnosis and surgery.

Assessment of cardiac volumes, mass, and function

There is abundant evidence linking ventricular function, volumes, and mass with prognosis in coronary artery disease and other cardiac disease. However, current techniques are not ideal. Radionuclide techniques suffer from a radiation burden which is best avoided if possible, and echocardiography is limited by technical issues such as image quality and acoustic windows which hinder quantification. These techniques have now been shown to be less accurate and reproducible than CMR, ${ }^{11}$ which has become a new gold standard. The interstudy reproducibility of CMR is now recognised by pharmaceutical companies which are switching to CMR for phase 2 and 3 drug development studies in order to reduce the sample size of their studies (fig 4$),{ }^{11}$ which saves time on recruitment and reduces costs significantly. Comparisons of established techniques and CMR show that mean values in populations vary between techniques by reasonably small amounts, but that individual variation can be very substantial. ${ }^{12}$ This means that the techniques are not interchangeable for individuals. If clinical decisions are based on numerical thresholds therefore, CMR is the preferred technique. The acquisition of these parameters by CMR is fast on the most modern scanners and can be achieved in a few minutes. Analysis still remains an area requiring improvement, however, and many centres still perform this manually, but new software with highly automated analysis is now coming on to the market.

\section{Flow and shunts}

CMR has particular application for the measurement of flow in the cardiovascular system. The normal images obtained reflect the magnitude of the radio signals received, but for each pixel in the image, the relative signal phase can also be measured. The phase can be encoded to relate linearly with velocity, and hence produce two dimensional velocity maps exactly corresponding with the anatomy from the normal images. Because the area of a vessel can easily be measured, and the mean velocity within the vessel calculated, absolute measurements of instantaneous flow can be derived. When run in cine mode throughout the cardiac cycle, flow curves are generated, where the area under the curve represents true flow in the vessel through the cardiac cycle. This is very valuable in the non-invasive measurement of the 


\section{Key points}

CMR is a rapidly growing new speciality in cardiovascular medicine

- CMR uses radio waves to generate images and is very safe

- CMR is versatile and can assess anatomy, function, flow, and several tissue characteristics

- The latest generation of CMR scanners allow real time scanning

- Applications of CMR in coronary artery disease are increasingly useful clinically

- The high reproducibility of CMR functional measurements has found significant research application in research into mechanisms of disease, and new drugs

pulmonary to systemic flow ratio (Qp:Qs) in cardiac shunting (fig 5), and in a number of other clinical scenarios.

\section{Valvar heart disease}

Echocardiography is ideal for investigating most valvar disease, but CMR has some particular uses. In valvar regurgitation, echo identifies the lesion, but it is not straightforward to ascertain the severity of the regurgitant flow. With CMR, the regurgitant flow can be measured directly using reverse flow measurement in diastole with an imaging plane placed just downstream of the aortic and pulmonary valve. ${ }^{13}$ Extension to assessment of mitral regurgitation involves the subtraction of aortic flow from true left ventricular stroke volume measured using the multislice technique. The technique is especially useful when surgery is being considered and clinical and echo results are not concordant, or there is doubt. For valvar stenosis, Doppler is more reliable for assessment and CMR is required less often, but again is helpful when conventional assessment has failed, such as in aortic stenosis with a distorted valve when a catheter cannot be passed across the valve. Experience suggests that CMR is a useful alternative to echocardiography for both stenosis and regurgitation, when flow across the valve is very eccentrically orientated.

\section{Pericardium}

The thickness of the pericardium is a good guide to the presence of constriction. This can be readily measured using CMR, although the thickness is slightly greater than expected from pathological studies, and this results from an effect known as chemical shift, caused by fat overlying the thin fibrous pericardial tissue. The normal CMR thickness of pericardium is therefore quoted as less than $4 \mathrm{~mm}$. The pericardium appears black on CMR because of its low water content, although in acute inflammation it can enhance with gadolinium and appear bright. The thickness of the pericardium needs to be distinguished from any pericardial effusion. Pericardial effusion is commonly black on spin echo images, but bright on gradient echo cines, and this is a useful means of differentiation. Pericardial calcification is not well seen by CMR, as calcium appears black, and therefore may simply appear as a localised area of pericardial thickening. CT is the best technique for showing calcium. Diastolic flow abnormalities can be demonstrated using velocity mapping, which can aid in diagnosis.

\section{Cardiomyopathy}

The contributions of CMR to the assessment of cardiomyopathy are in order of importance: arrhythmogenic right ventricular cardiomyopathy (ARVC), thalassaemia, hypertrophic cardiomyopathy, and sarcoidosis.
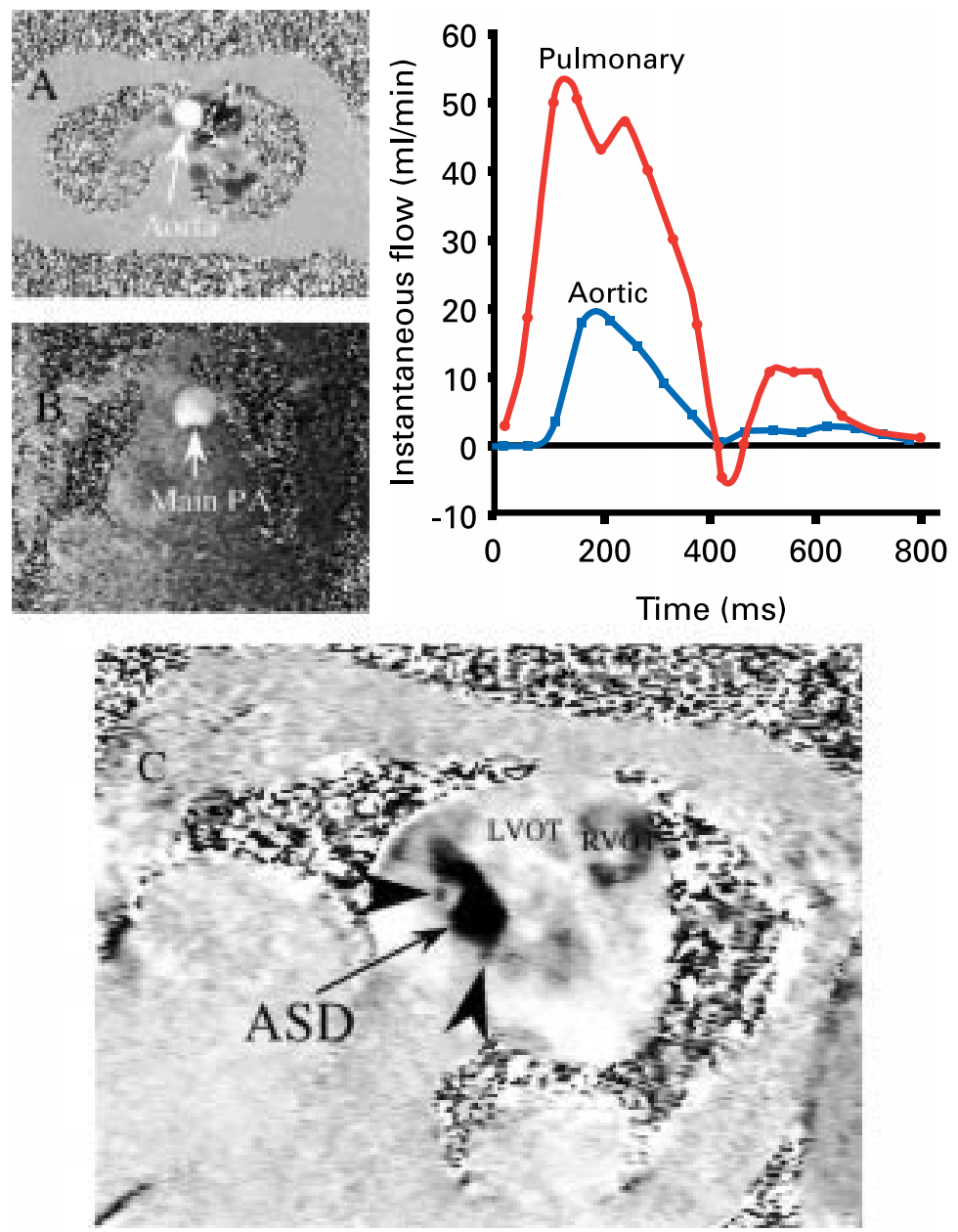

Figure 5. CMR of a patient with an atrial septal defect (ASD) in which conflicting echocardiographic and catheterisation results as to the size of the shunt had been obtained. (A) Velocity map (single frame) in a transverse plane for measurement of flow in the aorta; (B) velocity map (single frame) in a coronal plane for measurement of flow in the main pulmonary trunk (main PA); and graph of systemic and pulmonary flow plotted against time. The flow is calculated from the area under each curve, and the Qp:Qs ratio was 4:1 indicating a very substantial shunt.

(C) Phase velocity map of the ASD viewed from the right atrium in an oblique transaxial plane (RVOT, right ventricular outflow tract; LVOT, left ventricular outflow tract). The large ASD is seen as black pixels due to transseptal flow into the right atrium, and has a maximum diameter of $3.5 \mathrm{~cm}$ and an area of $5.6 \mathrm{~cm}^{2}$. The arrow heads indicate adjacent fenestrations. Reproduced from Taylor et al, J Cardiovasc Magn Reson 1999;1:43-7, with permission of the publisher. 
ARVC is a complex disorder which typically affects young men causing ventricular tachycardia of right ventricular origin, and sudden death. It is familial and gene abnormalities resulting in ARVC have been discovered. The disease presents with abnormalities of the right ventricle $(15 \%$ also have left ventricular involvement) and these are best delineated by CMR, as they can be subtle and localised (fig 6). ${ }^{14}$ The late stages with a large poorly functioning right ventricle and widespread fat infiltration are easy to identify, but in the early stages abnormalities typically include discrete areas of fat infiltration, regional hypokinesia, localised myocardial thinning, and abnormal trabeculations. Considerable experience is required to differentiate these findings from normal variants, and in particular the normal patterns of epicardial fat distribution associated with the right coronary and left anterior descending coronary arteries must be understood. It is important to not overdiagnose the condition because of the significant effects on the patient's insurance status and the psychological wellbeing of the rest of the family. CMR is the technique of choice for the assessment of ARVC, but cases are best assessed in experienced CMR centres seeing several potential early cases per week.

The assessment of myocardial iron overload has been problematic in clinical practice, but recently a T2^ $\mathrm{CMR}$ technique has been

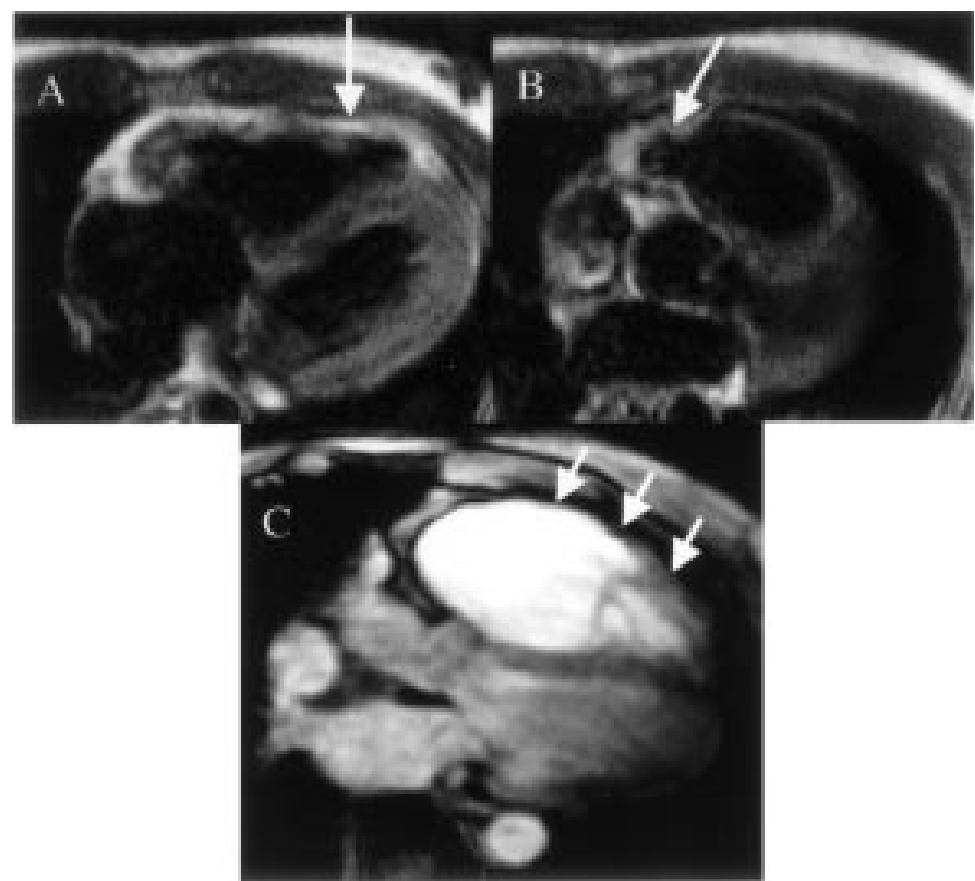

Figure 6. CMR features of arrhythmogenic right ventricular cardiomyopathy (ARVC). (A) A mid-ventricular transaxial plane in a patient with early disease who presented with non-sustained ventricular tachycardias of left bundle branch block morphology. The vertical arrow indicates an area of high signal in the right ventricular wall which represents fatty replacement of the myocardium. (B) In the same patient, the angled arrow shows a slightly higher transaxial plane with a bulge in the medial portion of the right ventricular outflow tract. (C) In a different patient, end stage disease is shown in the transaxial plane, with a very large right ventricle pushing the left ventricle posteriorly, and a thinned right ventricular free wall (short arrows). In general, ARVC is best diagnosed in its early stages when regional wall motion abnormalities in the right ventricle are present. In the patient in $A$ and $B$, the region of fatty infiltration showed dyskinesia during systole, greatly increasing confidence in the diagnosis.

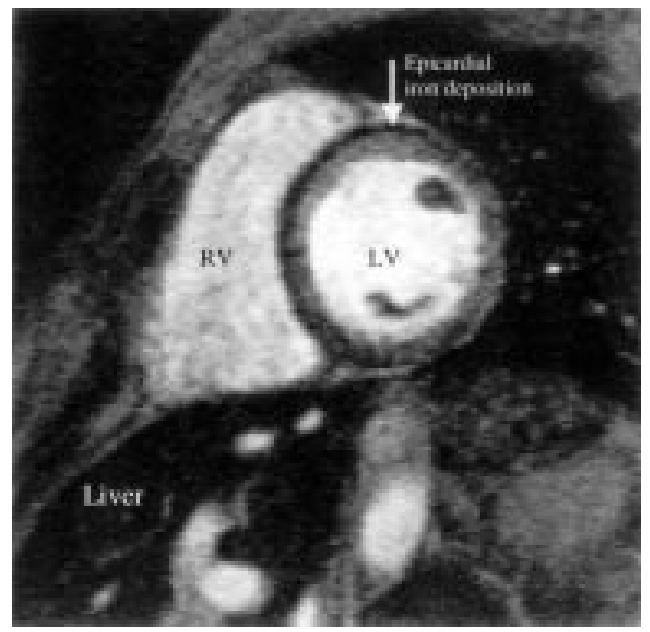

Figure 7. Example of iron deposition in a patient with thalassaemia. The dark epicardial rim of iron is arrowed. Note that liver deposition is very heavy, and the liver is therefore black. The signal loss occurs because of disturbances in the relaxation parameters of the tissues brought about by the iron causing alterations in the local magnetic field. There is very poor correlation between iron deposition in the liver and the heart, which prevents adequate management of the cardiac complications of myocardial iron overload (arrhythmia, heart failure, and death) from liver biopsy results. RV, right ventricle; LV, left ventricle. Reproduced from Rajappan et al, Eur $J$ Heart Failure 2000;2:241-52, with permission of the publisher.

established which allows reproducible quantification of the iron concentration (fig 7). ${ }^{15}$ Results show that iron loading in the myocardium is not correlated with other tissues such as the blood and liver, and must be examined separately. $\mathrm{T} 2{ }^{\star}$ values below $20 \mathrm{~ms}$ indicate incipient heart failure, which is frequently precipitous, and requires urgent intensive chelation treatment. This technique is likely to reduce considerably the premature mortality from heart failure in thalassaemia which is the most common cause of death in these patients. The technique can also be used in other iron overload conditions such as haemochromatosis and sickle cell anaemia.

In hypertrophic cardiomyopathy, the diagnosis can usually be made by echocardiography, but there are circumstances when CMR is very useful. If the condition is suspected but not confirmed by echocardiography, CMR is ideal as a second line investigation. CMR also shows the distribution of hypertrophy very well, and is superior for quantification of myocardial mass. CMR appears to be more sensitive than echocardiography for the diagnosis of apical hypertrophic cardiomyopathy and can be considered early in the diagnostic work up of these patients. Finally, CMR is valuable for the assessment of septal ablation techniques in locating the size and extent of infarction and assessing rest and stress outflow tract gradients.

The final area where CMR has utility is in cardiac sarcoidosis. This is a rare condition but one which is important as arrhythmias or ventricular dysfunction may be arrested with steroids. The myocardium appears speckled with CMR spin echo techniques, but enhancement occurs with gadolinium, and this makes infiltration or focal fibrosis more obvious. Little

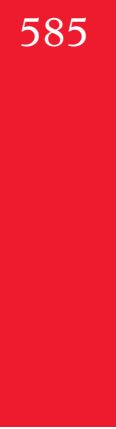


work has been published in this area, but in experienced centres, abnormal myocardial enhancement has been shown in a significant proportion of patients with known pulmonary disease.

\section{Coronary anomalies}

Coronary anomalies occur in approximately $1 \%$ of the population and are usually clinically silent. There are many anatomical variations which have been recorded and their definition requires not only the origin of the vessel, but also the proximal course. The importance of this condition lies in the occurrence of sudden death in patients who have a coronary artery passing between the great vessels (aorta and pulmonary artery). This may occur because of compression or kinking during exercise. Coronary ( $x$ ray) angiography commonly identifies the anomalous origin of the coronary artery, but is poor at defining the proximal course and the relation to the great vessels. Coronary $\mathrm{CMR}$ is now a robust technique for showing the origin and course of the proximal coronaries, and also the three dimensional relations of the great vessels. Several studies have shown that CMR is superior to $x$ ray angiography for this purpose. More recently, coronary CMR has been applied to patients with congenital heart disease in whom the incidence of coronary anomaly is up to $30 \%$, and defining the course by $x$ ray angiography is even more complex because of altered positions of the great vessels and ventricles. ${ }^{16}$ CMR was shown to be superior to $x$ ray angiography in defining the anomalies, and this is important for operative planning - for example, with vessels that cross in front of the right ventricular outflow tract.

\section{Indications close to clinical reality}

\section{Detection of myocardial infarction}

Recently the technique of late enhancement with gadolinium contrast agent has been described, in which imaging of the heart is performed 15 minutes after an intravenous injection of gadolinium (fig 8). The gadolinium concentrates in the necrotic (acute infarction) or scar tissue (chronic infarction) because of an increased partition coefficient, and the infarcted area becomes bright. ${ }^{17}$ There is very close correlation of the volume of signal enhancement and infarct size in animal experiments of acute infarction. The technique has high resolution, and can define the transmural extent of necrosis and scar for the first time in vivo. Although the technique has been recently developed, it has obvious applications in defining whether infarction has actually occurred in borderline cases; it also has the major advantage of not being subject to a time window of positivity like cardiac enzymes and pyrophosphate imaging. In addition, a different technique known as early enhancement (at 1-2 minutes after gadolinium injection) can be used to define the extent of microvascular obstruction in acute infarctions, which again is now possible for the first time clinically. Microvascular obstruction has already been shown to predict remodelling and adverse cardiac events after infarction. It is likely these techniques will rapidly find their way into clinical practice in centres with CMR available.

Assessment of myocardial viability

The technique of late enhancement has clinical application to the assessment of viability which is under intense current scrutiny. In a recent study, the simple morphological measure of the percentage transmural replacement of normal myocardium by scar has been shown to be a powerful predictor of post-revascularisation contraction recovery. Segments with less than $50 \%$ transmural replacement improved function, while those with higher grades of transmural replacement failed to improve. ${ }^{18}$ There are many clinical advantages of this technique-it is morphologically based, making analysis straightforward, and there is no radiation burden. It is anticipated that the late enhancement technique will make substantial
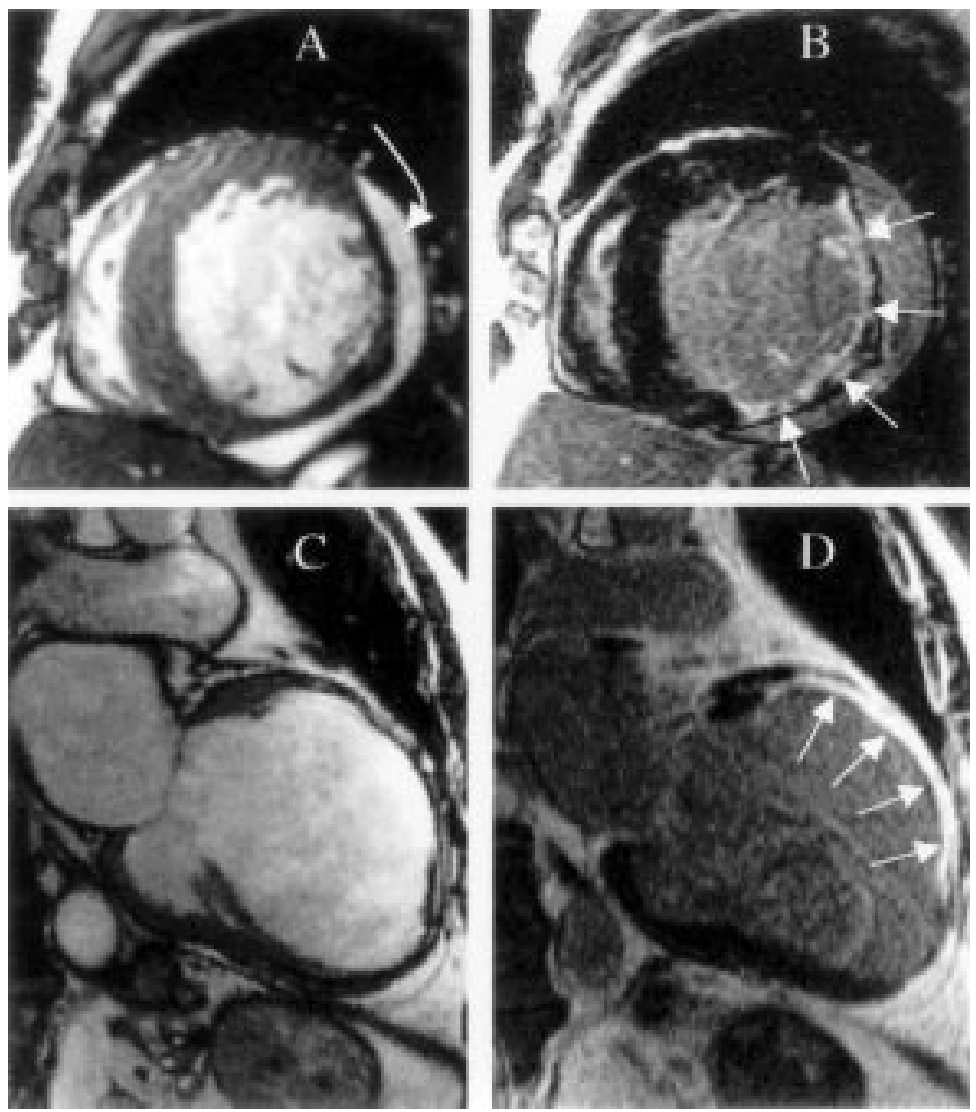

Figure 8. Myocardial infarction can be identified and viability can be determined using the late enhancement pattern after intravenous gadolinium. This figure shows two different patients, with the top row showing a lateral infarction, and the bottom row showing an anterior infarction. The left column ( $A$ and $C$ ) shows single frames from gradient echo cines, and the right column ( $B$ and $D$ ) shows the gadolinium enhanced images. The bright areas in B and D are infarcted tissue which is brightly enhanced (straight arrows). The infarct in the short axis plane (B) extends from the inferior wall to the anterolateral wall, and is mainly non-transmural. In particular, at 4 o'clock a significant rim of viable epicardial tissue is present and wall thickness is preserved. However, wall thinning has occurred elsewhere where the transmural

extent of infarction is greater. The infarct in the anterior wall of the vertical long axis plane (D) is transmural, however, and considerably greater thinning and ventricular remodelling has taken place. This technique allows transmural high resolution

infarct depiction in-vivo for the first time. The curved arrow shows a pericardial effusion. Reproduced from Rajappan et al, Eur J Heart Failure 2000;2:241-52, with permission of the publisher. Images courtesy of R Kim and R Judd. 
clinical impact in the management of infarction and its sequelae.

Dobutamine stress testing

Although it was first shown that dobutamine CMR was useful for detection of ischaemia in coronary artery disease a decade ago, it was not until its application in conjunction with modern scanners that the technique has achieved clinical validity. Comparisons with stress echocardiography have shown significantly improved diagnostic parameters with CMR which are related to greatly improved results from those patients in whom echocardiographic image quality was suboptimal or poor. ${ }^{19}$ Results from centres with experience approaching 1000 patients have begun to establish this technique, and, where available, it can be considered as a first line approach if acoustic windows are limited.

\section{CMR techniques in development}

Myocardial perfusion

There are approximately six million perfusion studies performed worldwide annually, and all currently involve exposure to ionising radiation. A safer technique would be medically welcome, providing it can maintain the same valuable diagnostic and prognostic information and is cost effective. Perfusion CMR may be a contender for this role (fig 9). The CMR technique is fast and simple with a baseline first pass perfusion study and a repeat study during adenosine stress. Gadolinium is used as the contrast agent with a peripheral intravenous injection. Areas of reduced perfusion show as dark areas in the myocardium surrounded by normal enhancing areas. One of the advantages of perfusion CMR is high resolution, which allows the visualisation of subendocardial perfusion defects in vivo for the first time. Quantitative analysis tools have been developed which examine the slope of signal increase during the first pass and the myocardial perfusion reserve index. Analysis on a pixel by pixel basis has allowed the generation of parametric perfusion maps and bullseyes, which are very similar to scintigraphy and aid immediate clinical recognition. ${ }^{20}$ Other advantages of perfusion CMR include the speed of the examination (one hour compared with typically 2-6 hours for a nuclear examination), and the easy combination with other techniques such as late enhancement (which depict areas of scar, and is available shortly after the perfusion study), as well as regional wall contraction.

\section{Atherosclerotic plaque}

Further down the road for clinical use is the technique of plaque imaging. CMR can be used to depict plaques and interrogate their lipid constituents as well as the integrity of the fibrous cap, which are factors that determine the propensity to plaque rupture and thrombus formation. T2 imaging has proved so far to be the most helpful, and validation of results

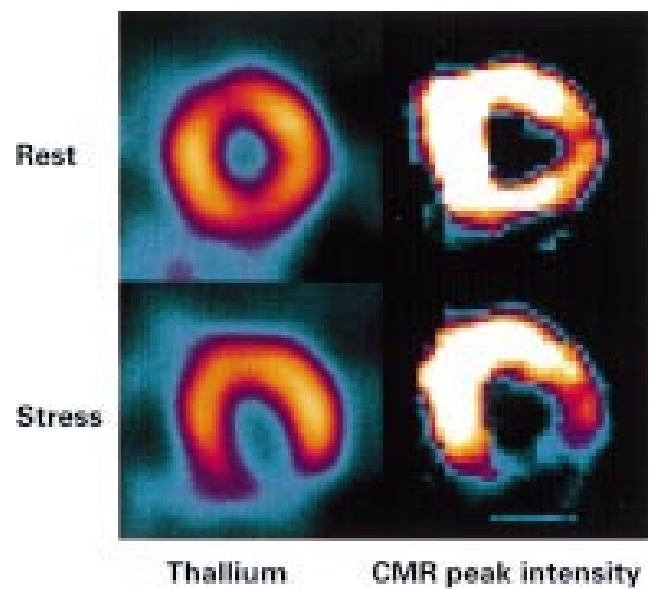

Figure 9. Comparison of thallium (left column) and CMR (right column) perfusion imaging in a patient with right coronary stenosis and inferior reversible ischaemia. The CMR images are parametric maps which are colour coded to appear similar to the thallium scan. Each pixel in the image represents the relative time to peak enhancement and bright colour indicates faster contrast wash-in and therefore better perfusion. The defect in the CMR scan is very similar in size and intensity to the thallium scan.

against pathology has been achieved in the aorta and carotids. Latest results have also shown plaques in the coronary arteries (fig 10). ${ }^{21}$ In addition, transoesophageal and intravascular imaging have now become available, and there is currently intense interest in comparison of these techniques with intravascular ultrasound. We will not know for some years the clinical impact of these techniques.

\section{Coronary imaging}

Much work has been devoted to the noninvasive assessment of the coronary artery lumen by CMR. ${ }^{22}$ There have been tremendous improvements in techniques since the

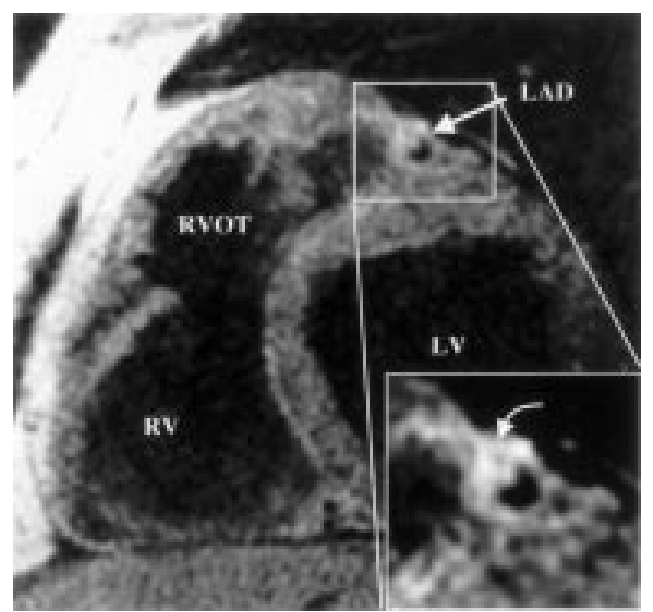

Figure 10. Coronary wall imaging in a patient with mild to moderate proximal left anterior descending (LAD) coronary artery stenosis. The enlargement shows eccentric vascular remodelling, as described by Glagov, with minor luminal encroachment. Note the dark area in the plaque (curved arrow). In validation studies, this represents the lipid core. Improvement in this type of imaging may lead to definition of the propensity to plaque rupture in vivo. $\mathrm{LV}$, left ventricle; RV, right ventricle; RVOT, right ventricular outflow tract. Reproduced from Fayad et al, Circulation 2000;102:506-10, with permission of the publisher. 


\section{Role of CMR in ischaemic heart disease}

- Accurate and reproducible ventricular volume and mass measurements in: -heart failure -hypertension -assessment of drug treatment -drug research

Detection of presence and extent of infarction

- Assessment of viability (transmural extent of infarction)

- Assessment of stress induced ischaemia (dobutamine wall motion CMR)

- Under development: -myocardial perfusion -coronary imaging -coronary flow -atherosclerosis imaging

first descriptions in 1993, most notably in the development of "navigators", which allow patients to breathe freely during the acquisition, with increased resolution and new sequences. ${ }^{23}$ Most recently three dimensional breath-hold techniques during contrast agent infusion have shown good results. It is likely that coronary CMR will prove useful in coronary artery disease at some point in the future, but currently its clinical robustness needs improvement, and the issue of preventing coronary motion during the acquisition has to be improved further. However, the success of the technique for coronary anomalies is established and vein graft imaging by CMR is now straightforward. What remains unclear in the longer term is the value of such a technique. With the major advances in depiction of ischaemia using perfusion and dobutamine CMR, there are rational arguments for suggesting that invasive angiography would simply move on to becoming a pre-interventional procedure rather than a diagnostic tool in most cases. The luminogram may not be the holy grail that it was thought to be 10 years ago.

\section{Education in CMR}

The rapid development of CMR techniques in cardiology makes the dissemination of the expertise in clinical practice a complex problem. It is likely that major cardiology institutions, with an interest in research, will want to consider the purchase of a dedicated CMR scanner in this decade. Speciality training has, however, barely begun, and there will be a shortage of expertise for some time; however steps in the right direction are happening with the new American College of Cardiology Core Cardiology Training Symposium (COCATS) guidelines for cardiology trainees in the USA recommending training in CMR. Fortunately international bodies such as the Society for
Cardiovascular Magnetic Resonance have looked ahead and have published credentialing guidelines to help develop adequate levels of training, whatever the professional background. ${ }^{24}$ This is new technology with workings that are not always intuitive, but we have found that newcomers with a good appreciation of cardiac physiology rapidly move through the learning curve.

1. Neubauer S, Horn M, Cramer M, et al. Myocardial phosphocreatine to ATP ratio is a predictor of mortality in patients with dilated cardiomyopathy. Circulation

1997;96:2190-6.

- This is a groundbreaking paper which shows that the phosphocreatine to ATP ratio in the failing heart is a better phosphocreatine to ATP ratio in the failing heart is a better
predictor of adverse outcome than other variables such as predictor of adverse outcome than other variables such as
ejection fraction and New York Heart Association functional class.

2. Kilner PJ, Yang GZ, Wilkes AJ, et al. Asymmetric redirection of flow through the heart. Nature 2000;404:759-61.

3. Strohm O, Kivelitz D, Gross W, et al. Safety of implantable coronary stents during $\mathrm{H}-1$ magnetic resonance imaging at 1.0 and 1.5T. J Cardiovasc Magn Reson 1999;1:239-45.

- There is much evidence now showing that all stents are safe to image. This paper was one of the first, and used laser techniques to show that no change in temperature laser techniques to show that no
occurs in the stent during CMR.

4. Francis JM, Pennell DJ. The treatment of claustrophobia during cardiovascular magnetic resonance; use and
effectiveness of mild sedation. $J$ Cardiovasc Magn Reson 2000;2:139-41.

5. Nienaber CA, von Kodolitsch Y, Nicolas V, et al. The diagnosis of thoracic aortic dissection by noninvasive imaging procedures. N Engl J Med 1993;328:1-9.

- One of the pioneering randomised trials comparing CMR with other imaging techniques for the diagnosis of acute aortic dissection. This showed that CMR was the most accurate technique to make the correct diagnosis.

6. Therrien J, Thorne SA, Wright A, et al. Repaired coarctation: a "cost-effective" approach to identify complications in adults. J Am Coll Cardiol 2000;35:997-

7. Hirsch R, Kilner PJ, Connelly M, et al. Diagnosis in adolescents and adults with congenital heart disease. Prospective assessment of the individual and combined roles of magnetic resonance imaging and transoesophageal echocardiography. Circulation 1994;90:2937-51.

- A seminal paper which showed the complementarity of transoesophageal echocardiography and CMR in the diagnosis of problems in congenital heart disease.

8. Owen RS, Carpenter JP, Baum RA, et al. Magnetic resonance imaging of angiographically occult runoff vessels in peripheral arterial occlusive disease. N Engl J Med 1992;326:1577-81.

9. Moody AR. Direct imaging of deep-vein thrombosis with magnetic resonance imaging. Lancet 1997;350:1073.

10. Frank H. Cardiac masses. In: Manning WJ, Pennell DJ, eds. Cardiovascular magnetic resonance. Philadelphia: Churchill Livingstone, 2001.

11. Bellenger NG, Davies LC, Francis JM, et al. Reduction in sample size for studies of remodelling in heart failure by the use of cardiovascular magnetic resonance. $J$ Cardiovasc Magn Reson 2000;2:271-8.

- An important paper showing that the improved interstudy reproducibility of CMR compared with echocardiography reproducibility of chestantial reductions in sample sizes required to allows substantial reductions in sample sizes required to prove a hypothesis of change in remodelling para
such as ventricular mass and diastolic volume.

12. Bellenger NG, Burgess M, Ray SG, et al on behalf of the CHRISTMAS Steering Committee and Investigators. Comparison of left ventricular ejection fraction and volume in heart failure by two-dimensional echocardiography, radionuclide ventriculography and cardiovascular magnetic resonance: are they interchangeable? Eur Heart $J$

2000;21:1387-96. - This study showed that in comparison with CMR as a gold of ejection fraction for individuals between techniques. This implies that management decisions for individuals based implies that management decisions for individuals based
on thresholds are best made using CMR, although in on thresholds are best made using CMR, althour
groups of sufficient size, the mean values for groups of sufficient size, the mean values for
measurements using all techniques are similar, and then only the sample size determines the ability to detect change between groups.

13. Dulce MC, Mostbeck GH, O'Sullivan M, et al. Severity of aortic regurgitation: interstudy reproducibility of measurements with velocity encoded cine MR imaging Radiology 1992;185:235-40. 
14. Blake LM, Scheinmann MM, Higgins CB. MR features of arrhythmogenic right ventricular dysplasia. $A J R$ $1994 \cdot 162 \cdot 809-12$.

15. Anderson LJ, Holden S, Davis B, et al. A novel method of cardiac iron measurement using magnetic resonance $\mathrm{T2}^{*}$ imaging in thalassemia: validation and clinical application [abstract]. Circulation 2000;102(Suppl II):403.

16. Taylor AM, Thorne SA, Rubens MB, et al. Coronary artery imaging in grown-up congenital heart disease: complementary role of MR and x-ray coronary angiography. Circulation 2000;101:1670-8.

17. Kim RJ, Fieno DS, Parrish RB, et al. Relationship of MRI delayed contrast enhancement to irreversible injury, infarct age, and contractile function. Circulation 1999;100:185-92.

18. Kim RJ, Wu E, Rafael A, et al. The use of contrast-enhanced magnetic resonance imaging to identify reversible myocardial dysfunction. N Engl J Med 2000;343:1445-53.

- Although this group from Chicago has published several validation papers showing the relation between infarct size and gadolinium uptake in animals, this paper extends the concept to viability in humans. In a myocardial segment, viability is preserved and post revascularisation improvement in function occurs, when transmural replacement by scar is less than $50 \%$. This is a simple high resolution technique which clearly shows the importance of residual epicardial muscle in viability.

19. Nagel E, Lehmkuhl HB, Bocksch W, et al. Noninvasive diagnosis of ischemia induced wall motion abnormalities with the use of high dose dobutamine stress MRI. Comparison with dobutamine stress echocardiography. Circulation 1999;99:763-70.

- A study of 208 consecutive patients undergoing catheterisation who also went on to have dobutamine echocardiography and CMR. CMR significantly outperformed echocardiography because of superior image quality in patients in whom echocardiographic image quality was poor.

20. Panting JR, Gatehouse PD, Yang GZ, et al. Echo planar magnetic resonance myocardial perfusion imaging Parametric map analysis and comparison with thallium SPECT. J Magn Reson Imaging 2001;13:192-200.

21. Fayad ZA, Fuster V, Fallon JT, et al. Noninvasive in vivo human coronary artery lumen and wall imaging using black-blood magnetic resonance imaging. Circulation 2000;102:506-10.

- First images of coronary wall plaque formation with external remodelling of the arterial wall (as described by Glagov), with only minor luminal encroachment.

22. de Feyter PJ, Nieman K, van Ooijen P, et al. Non-invasive coronary artery imaging with electron beam computed tomography and magnetic resonance imaging. Heart 2000;84:442-8.

23. Botnar RM, Stuber M, Danias PG, et al. Improved coronary artery definition with T2-weighted, free breathing, three dimensional coronary MRA. Circulation 1999:99:3139-48.

24. Pohost GM, Higgins CB, Grist TM, et al. Guidelines for credentialing in CMR (cardiovascular magnetic resonance). Society of Cardiovascular Magnetic Resonance (SCMR) clinical practice committee. J Cardiovasc Magn Reson 2000;2:233-4.

Essential reading for professionals of all backgrounds wishing to train in CMR, to learn of these multidisciplinary guidelines indicating the recommended three month training required in order to satisfy credentialing criteria in this new field.



ur 